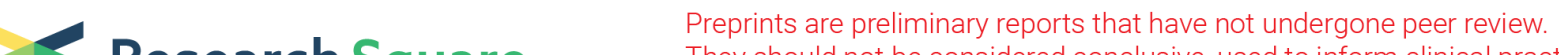 Research Square They should not be considered conclusive, used to inform clinical practice, or referenced by the media as validated information.
}

\section{Application of a self-designed inspiratory impedance threshold device in cardiopulmonary resuscitation in three porcine models of cardiac arrest}

Chao Li

Kunming Children's Hospital

Hai-Fei Gu

Kunming Children's Hospital

Xiao-Li He

Kunming Children's Hospital

Yu Zhang

Kunming Children's Hospital https://orcid.org/0000-0002-9863-4209

Jie-Qing Min

Kunming Children's Hospital

Lei Huang

Kunming Children's Hospital

Ming Zhang

Kunming Children's Hospital

Li-Ming Cheng

Kunming Children's Hospital

Ji-Lin Wu

Kunming Children's Hospital

Wen-Kang Du

Kunming Children's Hospital

Li Li (D lily20020302@hotmail.com )

https://orcid.org/0000-0003-3944-906X

\section{Research article}

Keywords: Cardiac arrest; cardiopulmonary resuscitation; Cardiac output; Ventricular fibrillation; Esophageal echocardiography

Posted Date: May 21st, 2019 
DOI: https://doi.org/10.21203/rs.2.9719/v1

License: (c) (1) This work is licensed under a Creative Commons Attribution 4.0 International License. Read Full License 


\section{Abstract}

Background: To evaluate and compare the efficiency of a self-designed inspiratory impedance threshold device in cardiopulmonary resuscitation (CPR) in the porcine models of cardiac arrest established by three approaches. Methods: Twenty healthy pigs were randomly assigned into the control group $(n=5)$, model $1(n=5)$, model $2(n=5)$ and model $3(n=5)$ groups. Following anesthesia induction, endotracheal tube was inserted and connected to the anesthesia machine. In the three model groups, pigs received intravenous injection of ketamine (model 1), MgSO4 (model 2) and $\mathrm{KCl}$ (model 3), and subsequently pig models of cardiac arrest were established. Manual closed-chest CPR was performed at $80 \mathrm{bpm}$ with the self-designed inspiratory impedance threshold device in the model groups and without this device in the control group. After 2-, 6- and 10-min CPR, the heart rate and hemodynamic parameters including arterial blood pressure, blood oxygen saturation, end-diastolic volume and cardiac output were quantitatively measured. The Esophageal echocardiography and blood-gas analyses were performed. Results: After $\mathrm{CPR}$, the mean arterial blood pressure, end-diastolic volume and cardiac output in three model groups were significantly higher compared with those in the control group (all $\mathrm{P}<0.05$ ). In model 2 group, the stroke volume, cardiac output, end-diastolic volume, SPO2 and PCO2 levels and blood-gas parameters were the highest among three model groups (all $\mathrm{P}<0.05$ ). Conclusions: The self-invented inspiratory impedance threshold device yields the highest efficiency in the porcine model of cardiac arrest established by intravenous infusion of MgSO4 by increasing the cardiac output during CPR compared with the remaining two pig models.

\section{Background}

Conventional manual cardiopulmonary resuscitation (CPR) can offer the blood supply to vital organs. Although CPR acts as the primary care for critically ill patients for years, the rescue rate of patients receiving conventional CRP is not satisfactory with a survival rate of lower than $7 \%$. CPR can exert the effect upon letting the blood flow from the heart to the periphery, which can be accomplished by sufficient venous blood flow to the chest following each cycle of manual compression. To enhance the survival and rescue rates of critically ill patients after CPR, novel mechanical instruments and devices have been explored and applied(1).

Among these techniques, active compression-decompression CPR is a technique which can transforms the thorax to a more efficient bellows. During the stage of decompression of CPR, the chest is highly pulled upward using a manual negative-pressure suction device, which is able to impose heavy negative pressure within the thoracic cavity and effectively promote the blood flow into the heart during the stage of decompression(2). During the conventional CPR, the blood flow from the peripheral veins to the heart significantly relies upon the status of natural chest wall recoil.

Previous investigations have demonstrated that complete airway occlusion during the stage of decompression can lead to high negative pressure within the thoracic cavity, accelerate the blood flow from the peripheral veins to the heart and considerably enhance the efficiency of conventional CPR(3). In 
previous researches(4), a newly-designed inspiratory impedance device was established to prevent the occurrence of inspiratory gas exchange throughout the stage decompression during CPR of the pressure within the thoracic cavity is lower compared with the atmospheric pressure.

In the present experiment, pig models were used as pigs carry similar size of hearts as human and are frequently used in previous CPR research. Healthy pigs were randomly assigned into the control group and three different models groups to establish cardiac arrest pig models via intravenous injection of ketamine, $\mathrm{MgSO}_{4}$ and $\mathrm{KCl}$, respectively. Subsequently, a self-designed inspiratory impedance threshold device (Patent registration No. CN 101697937 B) was developed and applied during CPR in the three animal models to evaluate the efficiency and safety, aiming to identify the self-designed device yields the highest efficiency in which pig model.

\section{Methods}

All animal experiments were performed with the approval of Animal Ethics Committee of Kunming Medical University. Animals were managed in accordance with Chinese Association for Laboratory Animal Sciences guidelines and current legal requirements. Our animal facilities meet the standards of Laboratory Animal-Requirements of Environment and Housing Facilities (GB 14925-2010). Twenty healthy pigs were randomly assigned into the control group $(n=5)$, model $1(n=5)$, model $2(n=5)$ and model $3(n=5)$ groups. Following anesthesia induction, endotracheal tube was inserted and connected to the anesthesia machine. In the three model groups, pigs received intravenous injection of ketamine (model 1), $\mathrm{MgSO}_{4}$ (model 2) and $\mathrm{KCl}$ (model 3), and subsequently pig models of cardiac arrest were established. Manual closed-chest CPR was performed at $80 \mathrm{bpm}$ with the self-designed inspiratory impedance threshold device in the model groups and without this device in the control group. After 2-, 6and 10-min CPR, the heart rate and hemodynamic parameters including arterial blood pressure, blood oxygen saturation, end-diastolic volume and cardiac output were quantitatively measured. The Esophageal echocardiography and blood-gas analyses were performed.

Self-designed inspiratory impedance threshold device

As illustrated in Figure 1, the self-designed cardiopulmonary resuscitation auxiliary device was equipped with an oxygen supply end (10) connected with an oxygen supply pipe on the bottom (5) with a cavity (4). It had an air supply exhalation end (9) connected with a patient's mask or a throat catheter and an evacuation end (6) connected with the atmosphere. A single control valve (2) and the first one-way valve (3) were set on the oxygen supply end (10). The second one-way valve (7) was designed on the evacuation end. One end of the column body (5) was fixed with the bent pipe (1). The vertical port (10) of the bent pipe (1) was downward to form an oxygen supply end connected with the oxygen supply pipe.

The other end of the column body (5) was fixed with a straight pipe (8) with a vertical upper port (6) and a vertical lower port (9), which was an air supply exhalation end to be connected with a mask or a laryngeal airway of a patient. The vertical upper port (6) of the straight pipe was an evacuation end, and the second 
one-way valve (7) was designed on the evacuation end to allow for exhalation, whereas prevent inhalation.

\section{Baseline data}

Twelve healthy miniature pigs ( 16 weeks old, indicating sexual maturity), both male and female, weighing $(28 \pm 5) \mathrm{kg}$ on average, were provided by the animal laboratory of Kunming Medical University and selected for subsequent experiment. The pigs used in the experiment were accustomed and fed in the laboratory for more than one day until all physiological parameters tended to be stable and be prepared for the subsequent experiment. The experimental animals were subject to dietary fasting but had free access to water on the night before surgery. The auxiliary device was designed based on the principle of negative pressure suction under this background. After completing the tracheal intubation, the selfdesigned device was connected. When the self-designed device was closed after each cycle of CPR was completed when the chest wall was pressed to the lowest point to occlude the flow of gas into the lungs. The effect of increasing the blood flow to the heart was achieved by using the principle of negative pressure suction generated by increasing the elastic retraction of chest wall.

\subsection{Establishment of pig models}

Anesthesia was used in all surgical interventions, all unnecessary suffering was avoided, and research was terminated if unnecessary pain or fear resulted in our pigs.

All pigs were subject to basic anesthesia via intramuscular administration of ketamine at a dose of 15 $\mathrm{mg} / \mathrm{kg}$, atropine $0.02 \mathrm{mg} / \mathrm{kg}$, and rimwest $0.2 \mathrm{mg} / \mathrm{kg}$, respectively. After the pig stood unstably, the animals were transferred and fixed on the operating table. Electrocardiogram was performed to measure the blood oxygen saturation $\left(\mathrm{SPO}_{2}\right)$. The auricular vein was cut open and supplemented with the balance salt solution at a dose of $20 \mathrm{ml} / \mathrm{kg}$. Intravenous injection of propofol $3 \mathrm{mg} / \mathrm{kg}$ and fentanyl $2 \mu \mathrm{g} / \mathrm{kg}$ were administered. Spontaneous breathing was retained and ID6.5-7.5 endotracheal tube with bursa was inserted with a depth of $16-18 \mathrm{~cm}$. After successful intubation, the breathing machine was connected to perform intermittent positive pressure mechanical ventilation (IPPV). The breathing parameters were adjusted with a breathing frequency of 15-20 times/min, a tidal volume of 8-10 ml/ $\mathrm{kg}$, and an inspiratoryto-expiratory ratio of 1:2. The end-expiratory carbon dioxide partial pressure $\left(\mathrm{ETPCO}_{2}\right)$ was kept at 35-40 $\mathrm{mmHg}$, which was connected to the Datex. Ohmeda monitoring machine. The femoral artery puncture was performed and was connected to the transducer on the monitoring machine to measure the dynamic blood pressure. Throughout the entire experiment, the esophageal TEE probe was inserted into the esophagus by approximately $30 \mathrm{~cm}$ to measure the cardiac output and returned blood volume of the experimental animals.

After the baseline data were recorded, intravenous injections were processed differently until the electrocardiogram hinted the signs of cardiac arrest. Ventricular fibrillation occurred prior to cardiac arrest. By observing the ventricular fibrillation waveform of electrocardiogram, ventricular fibrillation was defined when the arterial blood pressure was lower than $40 \mathrm{mmHg}$. The mechanical ventilation was 
terminated upon the incidence of ventricular fibrillation. CPR was initiated at the presence of cardiac arrest.

\subsection{CPR}

Under the same experimental conditions, the animals were randomly divided into the device group (group $\mathrm{I}, \mathrm{n}=6$ ) and non-device group (group II, $\mathrm{n}=6$ ). The depth of chest was $25 \%$ (approximately $5 \mathrm{~cm}$ ) of the anterior and posterior diameter of the chest. The compression frequency was 100 times/min to ensure the chest to fully rebound. The proportion of compression relaxation period was $50 \%: 50 \%$ to minimize the interruption as possible. CPR was terminated after chest compression endured for $4 \mathrm{~min}$. Cardiac arrest of all pigs in this experiment was induced by $5 \% \mathrm{KCl}$ solution, and no of them was successfully rescued after experiments. Carcass of pigs were handed over to the Animal Laboratory of Kunming Medical University for unified handling.

\subsection{Data collection}

Measurement of hemodynamic parameters: after the carbon dioxide was maintained within $35-40 \mathrm{mmHg}$ and hemodynamics remained stable, the heart rate (HR), mean arterial blood pressure (MAP), pulse blood oxygen saturation $\left(\mathrm{SPO}_{2}\right)$, end diastolic volume (EDV), cardiac output (CO) and alternative parameters were recorded as the baseline values. After the chest compression was started, the values of these parameters were recorded at $2 \mathrm{~min}, 4 \mathrm{~min}$ and $10 \mathrm{~min}$, respectively.

\subsection{Statistical analysis}

SPSS 19.0 software was utilized for statistical analysis (SPSS Inc., Chicago, IL). Measurement data were expressed as mean \pm standard deviation $(x \pm s)$. Hemodynamics and blood gases during CPR were analyzed with single factor ANOVA. A $P$ value of less than 0.05 was considered as statistical significance.

\section{Results}

Comparison of hemodynamic parameters

Prior to the induction of ventricular fibrillation, HR, systolic blood pressure (SP), diastolic blood pressure (DP) and mean arterial pressure (MAP) did not significantly differ among four groups (all $P>0.05$ ). Throughout the experiment, the SP was persistently elevated in the model 2 group $(P<0.05)$. At $2-, 6$ - and 10-min after CPR, the SP in the control group was significantly higher compared with those in the three model groups (all $P<0.05$ ), as illustrated in Figure 2A. During the entire experiment, the DP in the control and model 3 groups was continuously increased $(P<0.05)$, whereas no significant changes were noted in the model 1 and 2 groups (both $P>0.05$ ). At each time point following CPR, the DP in the control group was significantly higher than those in the three model groups (all $P<0.05$ ), as demonstrated in Figure $2 \mathrm{~B}$. During the entire experiment, the MAP in the model 2 and 3 groups tended to elevate, whereas that in the model 1 group tended to decline. At 2-, 6- and 10-min after CPR, the MAP in the control group was 
significantly higher than those in the three model groups (all $P<0.05$ ), as revealed in Figure $2 \mathrm{C}$. Regarding the HR, no statistical significance was observed at each time point after CPR between the control and three model groups (all $P>0.05$ ).

Comparison of esophageal ultrasound parameters

During the whole experiment, the stroke volume (SV) in the control and model 2 groups were significantly increased (both $P<0.05)$, whereas no statistical significance was noted in the model 1 group $(P>0.05)$. The SV tended to decline in the model 1 and 3 groups, as illustrated in Figure $3 \mathrm{~A}$. At each time point, the cardiac output (CO) was significantly increased in the control and model 2 groups (both $P<0.05$ ), whereas no statistical significance was observed in the model 1 and 3 groups $(P>0.05)$. The $C O$ in the model 1 and 3 groups tended to decline (Figure 3B). The ejection fraction in the control and model 1 groups tended to significantly increase (both $P<0.05$ ). The ejection fraction in the model 3 group tended to decline, and that was declined and then elevated in the model 2 group (Figure 3C).

Comparison of blood-gas parameters

In the model 1 and 3 groups, the $\mathrm{SPO}_{2}$ tended to decline, whereas that was increased in the model 2 group. The $\mathrm{SPO}_{2}$ was restored to the normal value at $10 \mathrm{~min}$ after $\mathrm{CPR}$ in the model 2 and 3 groups (Figure 4A). In three model groups, the $\mathrm{pH}$ values were significantly increased at each time point. At 10 min following CPR, the $\mathrm{pH}$ value was almost restored to normal in the model $2 \mathrm{group}$, whereas the $\mathrm{pH}$ at 10 min-CPR was significantly higher than the normal value in the model 3 group $(P<0.05$, Figure $4 \mathrm{~B})$. In three model groups, the $\mathrm{PCO}_{2}$ values were significantly declined at each time point after CPR. At 10 min after $\mathrm{CPR}$, the $\mathrm{PCO}_{2}$ was restored to normal in model 2 group, significantly higher than the normal value $(P<0.05)$ in the model 1 group, and considerably lower compared with the normal level in the model 3 group $(P<0.05)$, as demonstrated in Figure 4C. In the model 1 group, the $\mathrm{HCO}_{3}$ level was increased and subsequently decreased at each time point. In the model 2 group, the $\mathrm{HCO}_{3}$ level was persistently increased, whereas that was continuously declined in the model 3 group. At 10 min after $\mathrm{CPR}$, the $\mathrm{HCO}_{3}$ levels were almost restored to normal in the model 1 and 2 groups (Figure 4D). At 10 min after CPR, the $\mathrm{TCO}_{3}$ was almost restored to normal in the model 2 group. The changes of $\mathrm{TCO}_{3}$ were similar to those of $\mathrm{HCO}_{3}$. Identical variations were noted in terms of Beecf in three model groups (Figure 5).

In the model 1 group, the blood urea nitrogen (BUN) level was enhanced and then decreased at each time point. In the model 2 group, the BUN level tended to decline and subsequently elevate, whereas that was continuously declined in the model 3 group. At 10 min after CPR, the BUN levels were almost restored to normal in the model 1 and 3 groups (Figure 6A). At each time point, the white blood cell (WBC) count was increased in the model 1 group, the WBC did not significantly change in the model 2 group $(P>0.05)$, and that tended to decline in the model 3 group. At 10 min-CPR, the WBC was considerably lower than the normal value in the model 3 group $(P<0.05)$ (Figure 6B). In the model 1 group, the red blood cell $(\mathrm{RBC})$ count was increased and then decreased, that was decreased and subsequently increased in the model 2 group and that tended to decline in the model 3 group. In the model 3 group, the RBC was significantly 
lower than the normal at $10 \mathrm{~min}$ after CPR $(P<0.05)$ (Figure 6C). Similar outcomes were obtained for the hemoglobin (HGB) levels in three model groups. In the model 1 and 3 groups, the hematocrit (Hct) tended to decline and that was elevated in the model 2 group. At 10 min after CPR, the Hct was significantly lower than the normal value in the model 3 group $(P<0.05$, Figure 6D). In the model 1 group, the Na level was initially declined and then increased at each time point, that was elevated and then decreased in the model 2 group, and that was increased in the model 3 group. The Na values were restored to normal at each time point after CPR in three model groups (Figure 7A). In the model 1 group, the Na value was restored to normal at 10 min after $\mathrm{CPR}$, that was evidently higher in the model 2 group and significantly lower than the normal values in the model 3 group (both $P<0.05$ ). In the model 1 group, the $\mathrm{K}$ level was initially increased and then decreased at each time point, and that was declined in the model 2 and 3 groups (Figure 7B). In the model 1 group, the $\mathrm{Cl}$ level was initially decreased and then increased at each time point and that was decreased in the model 2 group, whereas persistently increased in the model 3 group. At each time point after $\mathrm{CPR}$, the $\mathrm{Cl}$ levels were significantly higher compared with the normal levels in three model groups (all $P<0.05$, Figure $7 \mathrm{C}$ ). In the model 1 and 2 groups, the Glu values tended to elevate and that was declined in the model 3 group. At 10 min after CPR, the Glu values were significantly higher than the normal levels in the model 1 and 2 groups, and that was evidently lower compared with the normal level in the model 3 group (all $P<0.05$, Figure 7D).

\section{Discussion}

In this experimental protocol, three animal models of cardiac arrest were established by intravenous injection of ketamine (model 1), $\mathrm{MgSO}_{4}$ (model 2) and $\mathrm{KCl}$ (model 3), respectively. After the establishment of three animal models, routine blood test parameters were quantitatively measured and statistically compared among three models. The detection outcomes demonstrated that the model 3 , which was established by intravenous administration of $\mathrm{KCl}$ was the optimal pig model for subsequent $\mathrm{CPR}$ experiment.

The variations of passive chest wall recoil and subsequent intra-thoracic pressure decide the blood return to the lungs and heart in standard CPR. Closed chest standard CPR without vasopressors cannot maintain minimal levels of vital organ blood flow required for life sustain(5-8). The results from this study demonstrate that intermittent airway occlusion with self-designed inspiratory impedance threshold device during the decompression phase in CPR enhanced the blood return to the heart and lungs and significantly enhance the blood return to vital organs. The application of the self-designed inspiratory impedance threshold device enhanced the efficiency of standard CPR, increased the cerebral blood flow and elevated the myocardial perfusion.

In this investigation, the hemodynamic parameters significantly differed among different groups, suggesting that application of the self-designed device can improve the efficiency of conventional CPR in pig models. By preventing the inspiratory gas exchange in standard CPR, an increased negative intrathoracic pressure was induced throughout the decompression phase of conventional CPR. 
Both hemodynamic parameters and blood flow data have validated the fundamental significance of increasing the blood return into the chest in conventional CPR. Although increasing cardiac arrest times can enhance the resistance to sufficient reflow to the vital organs(9), the use of the self-designed impedance valve can maintain relatively high SBP, DBP and MAP during the standard CPR in pig models, whereas the HR did not significantly differ between the control and model groups even with the application of the self-designed impedance valve, which is consistent with previous studies which compared the efficiency of conventional CPR with and without an impedance valve(10-13). With active decompression and use of the self-designed impedance valve, the left ventricular and brain blood flow can be approximately $55 \%$ and $125 \%$ of baseline levels(14). Previous clinical trials have demonstrated that for patients undergoing CPR, the mean blood pressure was 110/56 when CPR was performed with the impedance valve, which is significantly higher compared with 90/35 in patients receiving CPR without the application of the impedance valve(15).

The hemodynamic effect of the self-designed impedance valve during standard CPR is probably correlated with the arrest time, fluid status, endogenous hormone level, ventricular wall compliance and CPR duration, etc. Throughout conventional CPR, the compliance of the chest wall and the respiratory system significantly declines(16). The effect of the impedance valve is determined by multiple influencing factors during standard CPR, which could possibly explain why the impedance valve exerted a more significant effect when the cardiac arrest time was prolonged from 2 to $10 \mathrm{~min}$. A previous study has proven that the effect of the impedance valve was significant with a shorter arrest time, but the effect was less than those with longer arrest time(17). Earlier reports have identified that use of the selfdesigned impedance valve in the respiratory system prevented the ventilation by occluding the airflow during chest compression, leading to higher-than-normal arterial carbon dioxide values in the model groups(18). Besides, the blood gas parameters also supported the efficiency of the self-designed impedance valve in the model 3 group. At 10-min $\mathrm{CPR}$, the $\mathrm{SPO}_{2}, \mathrm{PCO}_{2}, \mathrm{HCO}_{3}$ and $\mathrm{BUN}$ levels, as well as Beecf value were almost restored within normal range in the model 3 group. In addition, the WBC, HGB, Hct and Glu levels were significantly lower than the normal values.

\section{Conclusions}

In this experiment, the pig models of cardiac arrest were established by three techniques and the comparative results demonstrated that the pig model established by intravenous injection of $\mathrm{KCl}$ was the optimal and ideal model for subsequent research. Besides, the supplementary use of the self-made impedance valve could significantly improve the blood return to the heart and lungs and evidently increase the cardiac output in the porcine models.

\section{Abbreviations}

CPR: cardiopulmonary resuscitation

IPPV: intermittent positive pressure mechanical ventilation 
ETPCO2: end-expiratory carbon dioxide partial pressure

TEE: Trans-Esophageal Echocardiogram

HR: heart rate

MAP: mean arterial blood pressure

SP02: pulse blood oxygen saturation

EDV: end diastolic volume

CO: cardiac output

SP: systolic blood pressure

DP: diastolic blood pressure

SV: stroke volume

BUN: blood urea nitrogen

WBC: white blood cell

RBC: red blood cell

HGB: hemoglobin

Hct: hematocrit

\section{Declarations}

Ethics approval and consent to participate

This study involved the use of animals and was approved by the Ethics Committee of Kunming Children's Hospital.

Consent for publication

Not applicable.

Availability of data and materials

The datasets used and analysed during the current study are available from the corresponding author on reasonable request.

Competing interests 
The authors declare that they have no competing interests.

Funding

The study was supported by grants from the National Natural Science Fund of China (No.81560262), the Basic Applied Study Planning Projects of Yunnan Province (No. 2018FB130), the Joint Special Fund for Basic Research from Yunnan Provincial Science and Technology and Kunming Medical University (No.2017FE467-149), and the High level Health and Family Planning Technical Personnel Training Projects of Yunnan Province (No. H-201626). These funding bodies provided financial support to our experimental materials, staff compensation and manuscript editing.

Authors' contributions

$\mathrm{CL}$ constructed the self-designed inspiratory impedance threshold device and animal models, and are main contributor in writing the manuscript; $\mathrm{HFG}, \mathrm{XLH}$ and $\mathrm{YZ}$ participated in the animal experiments, JQM, LH and MZ contributed in the data collection and results analysis; LMC, JLW and WKD contributed to results analysis and figure plotting; LL provided ideas and suggestions to the study. All authors read and approved the final.

Acknowledgements

Not applicable.

\section{References}

1. Aufderheide TP, Frascone RJ, Wayne MA, Mahoney BD, Swor RA, Domeier RM, et al. Standard cardiopulmonary resuscitation versus active compression-decompression cardiopulmonary resuscitation with augmentation of negative intrathoracic pressure for out-of-hospital cardiac arrest: a randomised trial. Lancet (London, England). 2011 Jan 22;377(9762):301-11.

2. Plaisance $P$, Lurie KG, Payen D. Inspiratory impedance during active compression-decompression cardiopulmonary resuscitation: a randomized evaluation in patients in cardiac arrest. Circulation. 2000 Mar 7;101(9):989-94.

3. Wolcke BB, Mauer DK, Schoefmann MF, Teichmann H, Provo TA, Lindner KH, et al. Comparison of standard cardiopulmonary resuscitation versus the combination of active compression-decompression cardiopulmonary resuscitation and an inspiratory impedance threshold device for out-of-hospital cardiac arrest. Circulation. 2003 Nov 4;108(18):2201-5.

4. Högler S, Sterz F, Sipos W, Schratter A, Weihs W, Holzer M, et al. Distribution of neuropathological lesions in pig brains after different durations of cardiac arrest. Resuscitation. 2010 Nov;81(11):1577-83.

5. Berg RA, Hilwig RW, Berg MD, Berg DD, Samson RA, Indik JH, et al. Immediate post-shock chest compressions improve outcome from prolonged ventricular fibrillation. Resuscitation. $2008 \mathrm{Jul} ; 78(1): 71-$ 
6. Plaisance P, Lurie KG, Vicaut E, Martin D, Gueugniaud P-Y, Petit J-L, et al. Evaluation of an impedance threshold device in patients receiving active compression-decompression cardiopulmonary resuscitation for out of hospital cardiac arrest. Resuscitation. 2004 Jun;61(3):265-71.

7. Staffey KS, Dendi R, Brooks LA, Pretorius AM, Ackermann LW, Zamba KD, et al. Liquid ventilation with perfluorocarbons facilitates resumption of spontaneous circulation in a swine cardiac arrest model. Resuscitation. $2008 \mathrm{Jul} ; 78(1): 77-84$.

8. Yannopoulos D, Matsuura T, McKnite S, Goodman N, Idris A, Tang W, et al. No assisted ventilation cardiopulmonary resuscitation and 24-hour neurological outcomes in a porcine model of cardiac arrest. Crit Care Med. 2010 Jan;38(1):254-60.

9. Idris AH, Becker LB, Ornato JP, Hedges JR, Bircher NG, Chandra NC, et al. Utstein-style guidelines for uniform reporting of laboratory CPR research. A statement for healthcare professionals from a task force of the American Heart Association, the American College of Emergency Physicians, the American College of Cardiology, the European Resuscitation Council, the Heart and Stroke Foundation of Canada, the Institute of Critical Care Medicine, the Safar Center for Resuscitation Research, and the Society for Academic Emergency Medicine. Writing Group. Circulation. 1996 Nov 1;94(9):2324-36.

10. Shultz JJ, Coffeen P, Sweeney M, Detloff B, Kehler C, Pineda E, et al. Evaluation of standard and active compression-decompression CPR in an acute human model of ventricular fibrillation. Circulation. 1994 Feb;89(2):684-93.

11. Neumar RW, Otto CW, Link MS, Kronick SL, Shuster M, Callaway CW, et al. Part 8: adult advanced cardiovascular life support: 2010 American Heart Association Guidelines for Cardiopulmonary Resuscitation and Emergency Cardiovascular Care. Circulation. 2010 Nov 2;122(18 Suppl 3):S729-67.

12. Koster RW, Sayre MR, Botha M, Cave DM, Cudnik MT, Handley AJ, et al. Part 5: Adult basic life support. Resuscitation. 2010 Oct;81(1):e48-70.

13. Berg RA, Hemphill R, Abella BS, Aufderheide TP, Cave DM, Hazinski MF, et al. Part 5: adult basic life support: 2010 American Heart Association Guidelines for Cardiopulmonary Resuscitation and Emergency Cardiovascular Care. Circulation. 2010 Nov 2;122(18 Suppl 3):S685-705.

14. Halperin HR, Lee K, Zviman M, Illindala U, Lardo A, Kolandaivelu A, et al. Outcomes from low versus high-flow cardiopulmonary resuscitation in a swine model of cardiac arrest. Am J Emerg Med. 2010 Feb;28(2):195-202.

15. Ali IS, Gandhi M, Finegan BA, Koshal A, Clanachan AS. Cardioprotection by activation of NO/cGMP pathway after cardioplegic arrest and 8-hour storage. Ann Thorac Surg. 1998 May;65(5):1303-9. 
16. Klouche K, Weil MH, Sun S, Tang W, Povoas HP, Kamohara T, et al. Evolution of the stone heart after prolonged cardiac arrest. Chest. 2002 Sep;122(3):1006-11.

17. Gazmuri RJ, Ayoub IM, Hoffner E, Kolarova JD. Successful ventricular defibrillation by the selective sodium-hydrogen exchanger isoform-1 inhibitor cariporide. Circulation. 2001 Jul 10;104(2):234-9.

18. Ayoub IM, Kolarova J, Kantola RL, Sanders R, Gazmuri RJ. Cariporide minimizes adverse myocardial effects of epinephrine during resuscitation from ventricular fibrillation. Crit Care Med. 2005 Nov;33(11):2599-605.

\section{Figures}

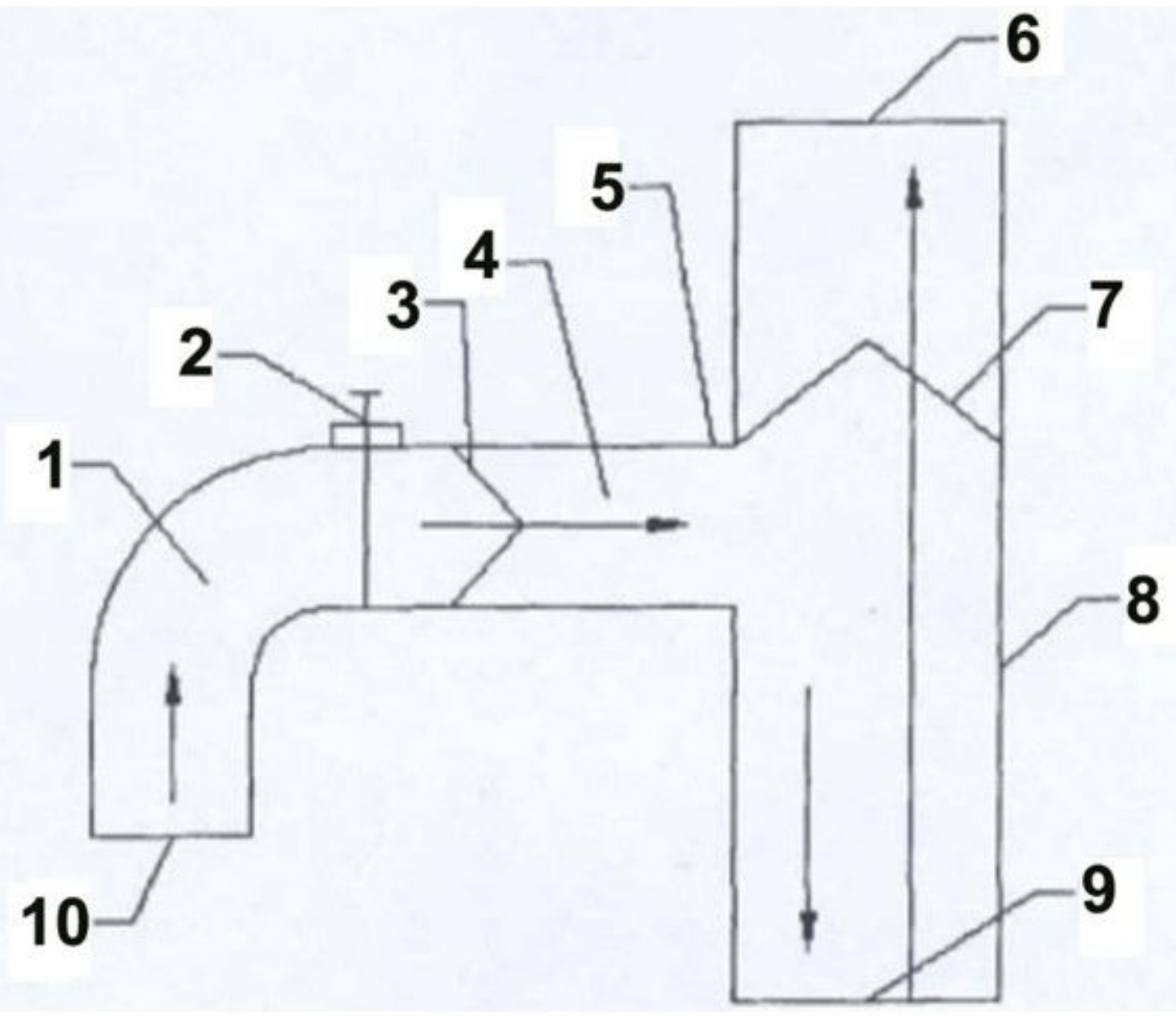

Figure 1

Structure of the self-designed inspiratory impedance threshold device. 1. Bent pipe; 2 . Control valve; 3. First one-way valve; 4 . Cavity; 5 . Oxygen supply pipe; 6 . Evacuation end; 7 . Second one-way valve; 8. Straight pipe; 9 . Vertical lower port; 10 . Vertical port. 

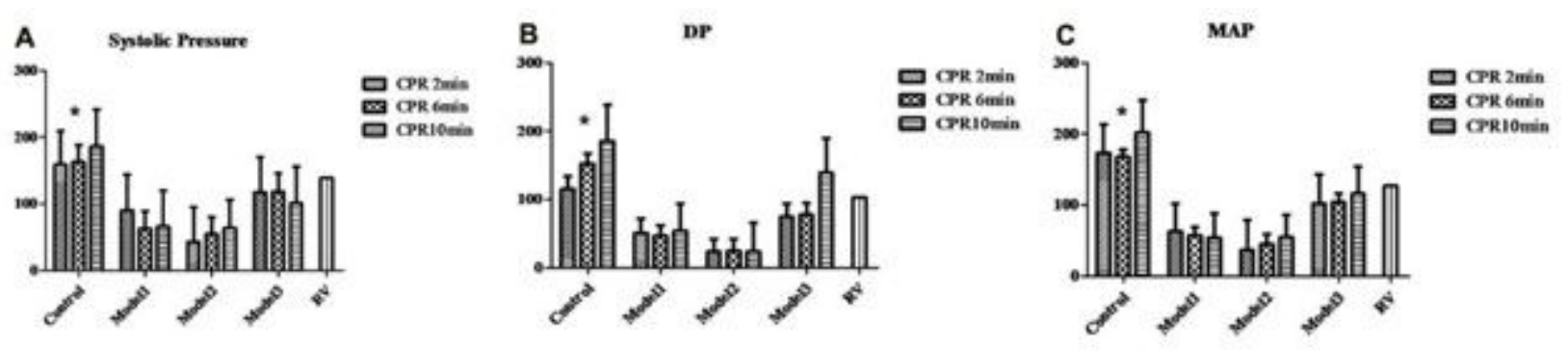

Figure 2

Comparison of hemodynamic parameters. A. SP was persistently elevated in the model 2 group $(P<0.05)$. At 2-, 6- and 10-min after CPR, the SP in the control group was significantly higher compared with those in the three model groups (all $\mathrm{P}<0.05$ ); B. DP in the control and model 3 groups was continuously increased $(P<0.05)$, whereas no significant changes were noted in the model 1 and 2 groups (both $P>0.05)$. DP in the control group was significantly higher than those in the three model groups (all $P<0.05$ ) at each time point following CPR; C. MAP in the model 2 and 3 groups tended to elevate, whereas that in the model 1 group tended to decline. At 2-, 6- and 10-min after CPR, the MAP in the control group was significantly higher than those in the three model groups (all $\mathrm{P}<0.05$ ).
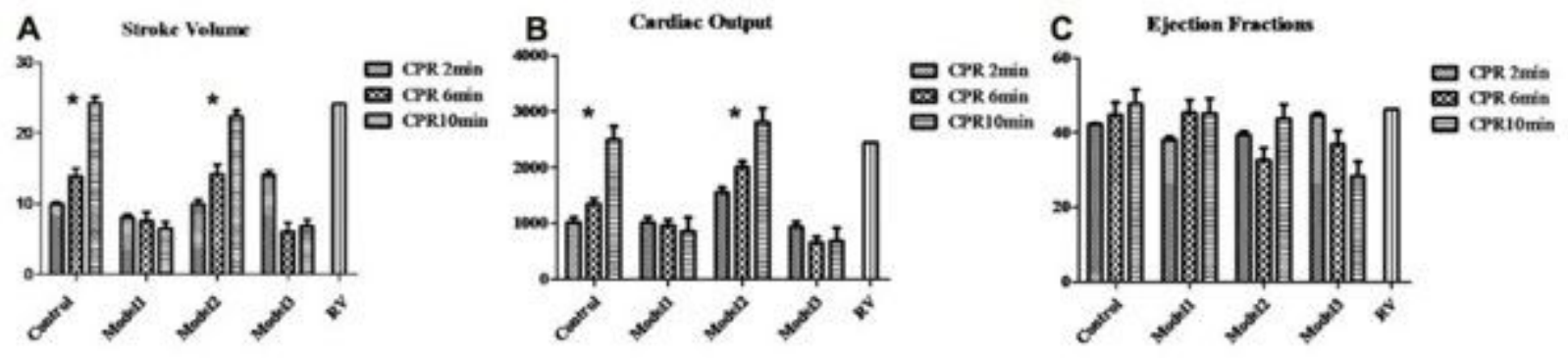

Figure 3

Comparison of esophageal ultrasound parameters. A. SV in the control and model 2 groups were significantly increased (both $\mathrm{P}<0.05$ ), whereas no statistical significance was noted in the model 1 group $(P>0.05)$. SV tended to decline in the model 1 and 3 groups; $B$. At each time point, $C O$ was significantly increased in the control and model 2 groups (both $\mathrm{P}<0.05$ ), whereas no statistical significance was observed in the model 1 and 3 groups $(P>0.05)$. $C O$ in the model 1 and 3 groups tended to decline; $C$. Ejection fraction in the control and model 1 groups tended to significantly increase (both $P<0.05$ ). The ejection fraction in the model 3 group tended to decline, and that was declined and then elevated in the model 2 group. 


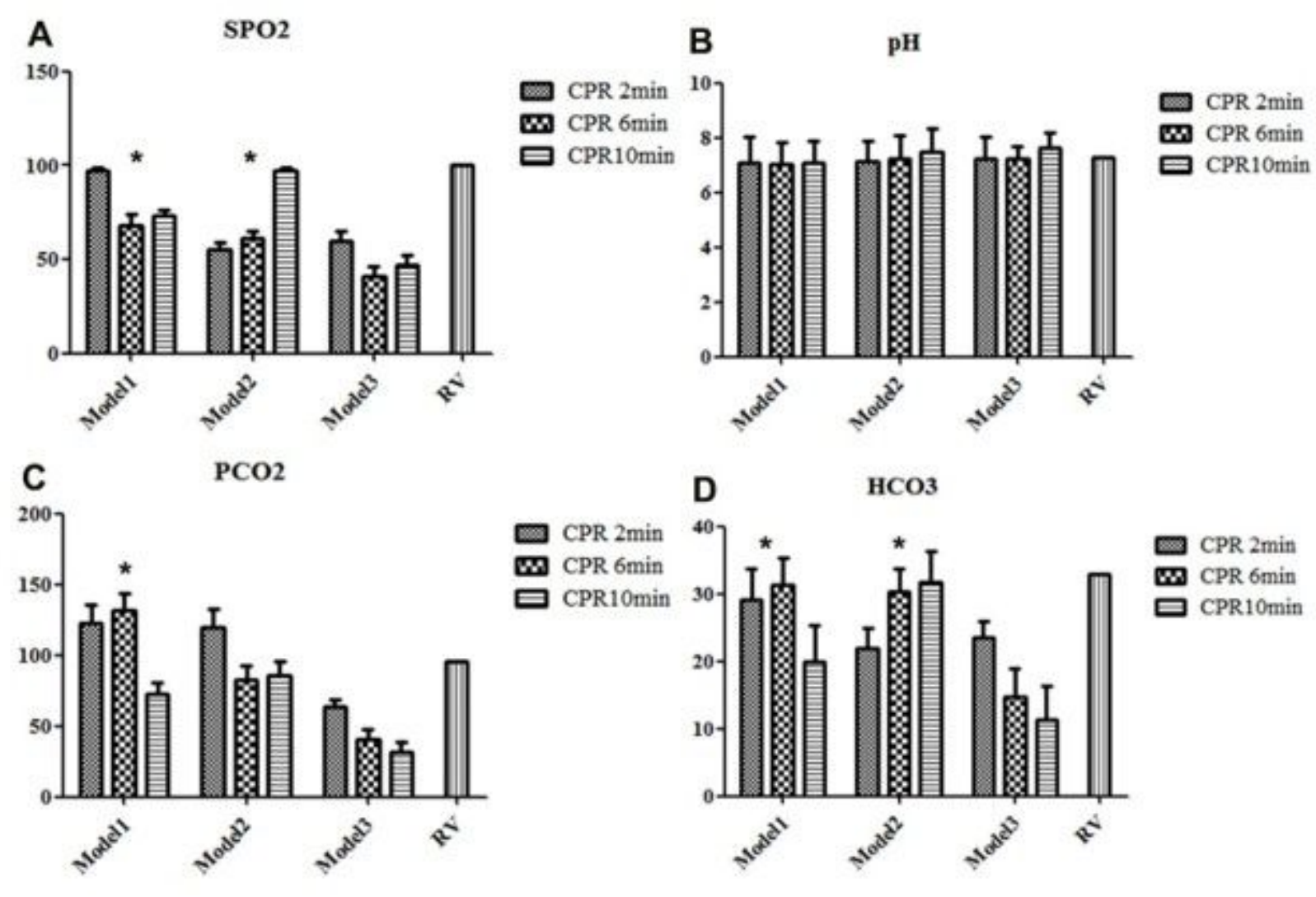

Figure 4

Comparison of SPO2, pH, PCO2 and $\mathrm{HCO} 3$ among different groups. A. In the model 1 and 3 groups, SPO2 tended to decline, whereas that was increased in the model 2 group. SPO2 was restored to the normal value at 10 min after CPR in the model 2 and 3 groups; $\mathrm{B}$. In three model groups, the $\mathrm{pH}$ values were significantly increased at each time point. At 10 min following $\mathrm{CPR}$, the $\mathrm{pH}$ value was almost restored to normal in the model 2 group, whereas the $\mathrm{pH}$ at 10 min-CPR was significantly higher than the normal value in the model 3 group; $\mathrm{C}$. In three model groups, the PCO2 values were significantly declined at each time point after CPR. At 10 min after CPR, the PCO2 was restored to normal in model 2 group, significantly higher than the normal value $(P<0.05)$ in the model 1 group, and considerably lower compared with the normal level in the model 3 group $(P<0.05)$; $D$. In the model 1 group, the HCO3 level was increased and subsequently decreased at each time point. In the model 2 group, the $\mathrm{HCO} 3$ level was persistently increased, whereas that was continuously declined in the model 3 group. At 10 min after CPR, the $\mathrm{HCO} 3$ levels were almost restored to normal in the model 1 and 2 groups. 


\section{Beecf}

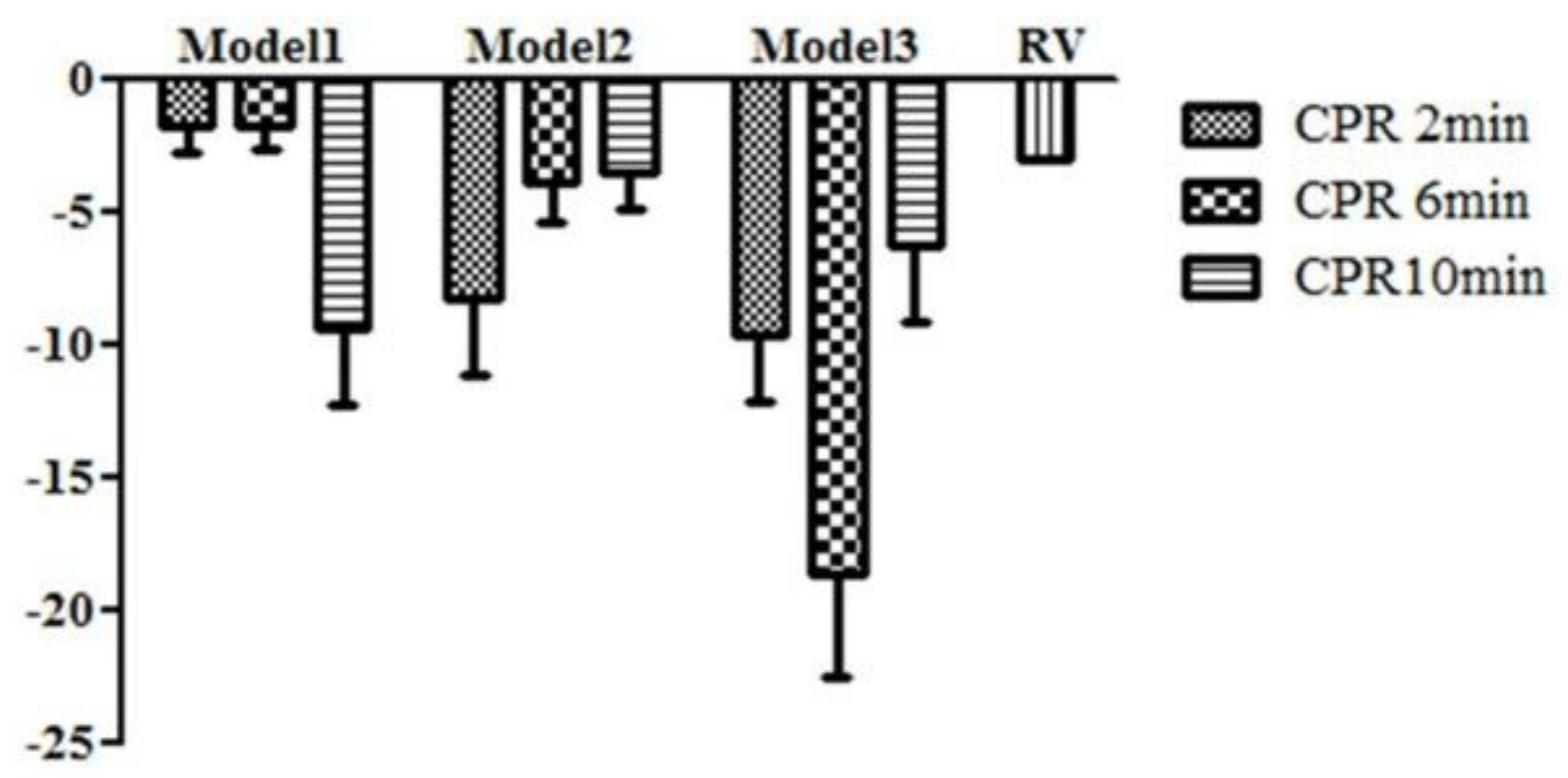

Figure 5

Comparison of Beecf values among different groups. Identical variations were noted in terms of Beecf in three model groups.

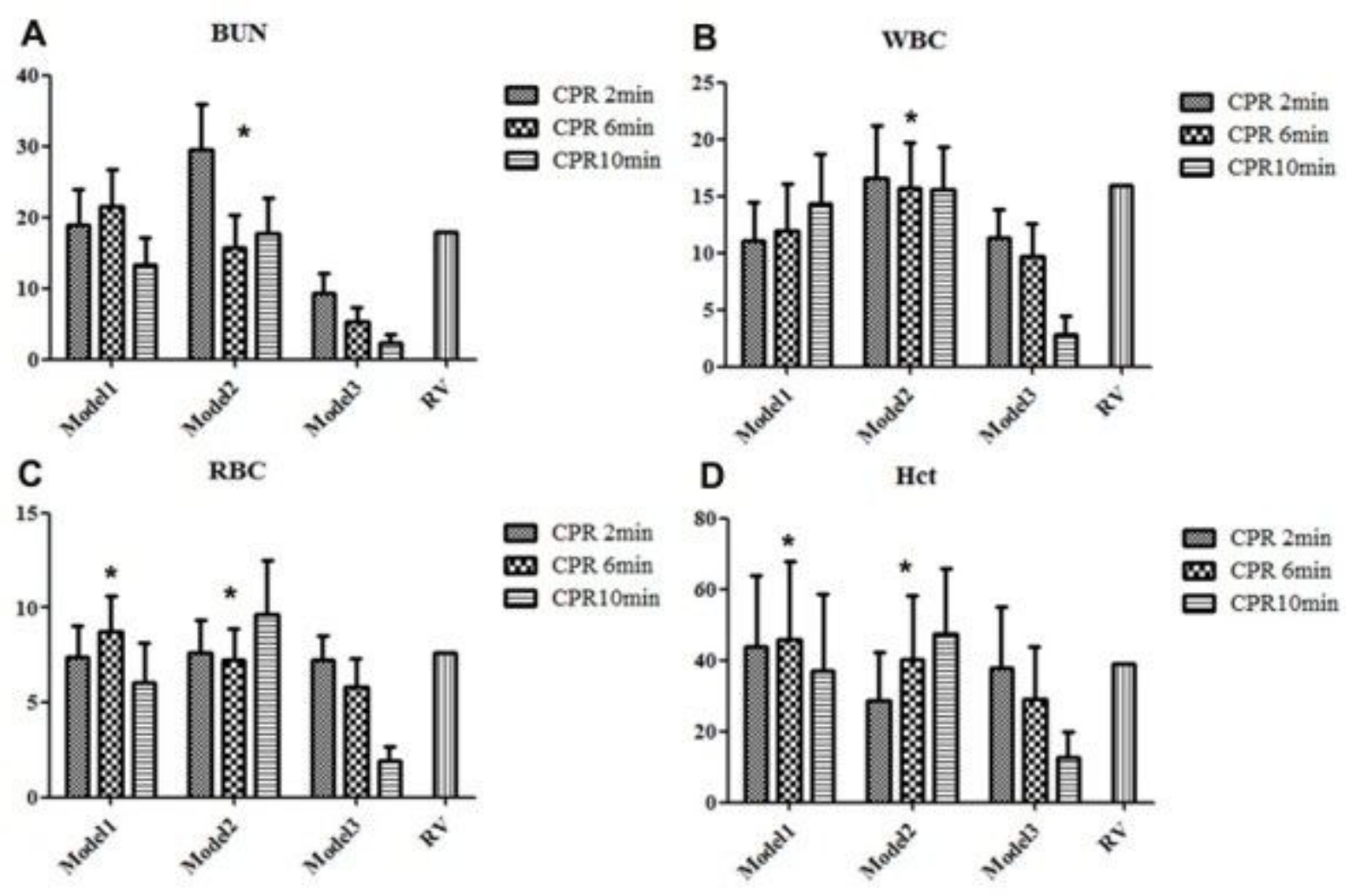

Figure 6 
Comparison of BUN, WBC, RBC and Hct among different groups. A. In the model 1 group, BUN level was enhanced and then decreased at each time point. In the model 2 group, the BUN level tended to decline and subsequently elevate, whereas that was continuously declined in the model 3 group. At 10 min after $\mathrm{CPR}$, the BUN levels were almost restored to normal in the model 1 and 3 groups; B. At each time point, WBC count was increased in the model 1 group, the WBC did not significantly change in the model 2 group ( $P>0.05)$, and that tended to decline in the model 3 group. At 10 min-CPR, the WBC was considerably lower than the normal value in the model 3 group $(P<0.05) ; C$. In the model 1 group, $R B C$ count was increased and then decreased, that was decreased and subsequently increased in the model 2 group and that tended to decline in the model 3 group. In the model 3 group, the RBC was significantly lower than the normal at 10 min after CPR $(P<0.05)$; D. In the model 1 and 3 groups, Hct tended to decline and that was elevated in the model 2 group. At 10 min after CPR, the Hct was significantly lower than the normal value in the model 3 group $(P<0.05)$.

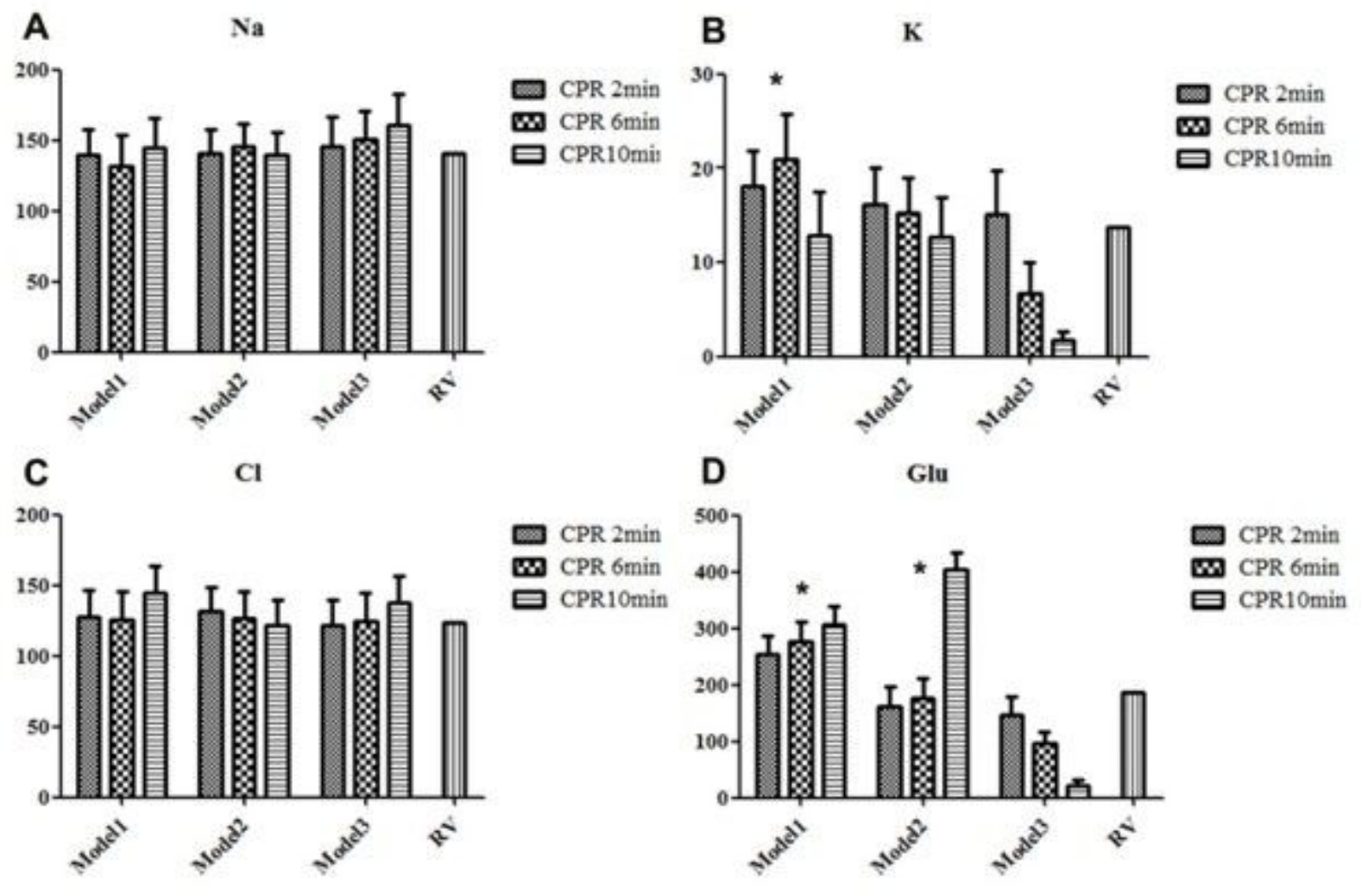

Figure 7

Comparison of $\mathrm{Na}, \mathrm{K}, \mathrm{Cl}$ and Glu among different groups. A. In the model $1 \mathrm{group}$, the $\mathrm{Na}$ level was initially declined and then increased at each time point, that was elevated and then decreased in the model 2 group, and that was increased in the model 3 group. The Na values were restored to normal at each time point after CPR in three model groups; $\mathrm{B}$. In the model 1 group, the $\mathrm{K}$ level was initially increased and then decreased at each time point, and that was declined in the model 2 and 3 groups; $\mathrm{C}$. In the model 1 group, the $\mathrm{Cl}$ level was initially decreased and then increased at each time point and that was decreased in the model 2 group, whereas persistently increased in the model 3 group. At each time point after $\mathrm{CPR}$, the $\mathrm{Cl}$ levels were significantly higher compared with the normal levels in three model groups 
(all $\mathrm{P}<0.05) ; \mathrm{D}$. In the model 1 and 2 groups, the Glu values tended to elevate and that was declined in the model 3 group. At 10 min after CPR, the Glu values were significantly higher than the normal levels in the model 1 and 2 groups, and that was evidently lower compared with the normal level in the model 3 group (all $\mathrm{P}<0.05$ ).

\section{Supplementary Files}

This is a list of supplementary files associated with this preprint. Click to download.

- supplement1.pdf 